\title{
SEQUENTIAL TESTS FOR NORMAL MARKOV SEQUENCE
}

\author{
R. M. PHATARFOD
}

(Received 14 August 1969)

Communicated by P. D. Finch

\section{Introduction}

This is a sequel to the author's (Phatarfod [9]) paper in which an analogue of Wald's Fundamental Identity (F.I.) for random variables defined on a Markov chain with a finite number of states was derived. From it the sampling properties of sequential tests of simple hypotheses about the parameters occurring in the transition probabilities were obtained. In this paper we consider the case of continuous Markovian variables. We restrict our attention to the practically important case of a Normal Markov sequence $X_{0}, X_{1}, X_{2}, \cdots$ such that

$$
X_{r}-m=\rho\left(X_{r-1}-m\right)+Y_{r}, \quad(|\rho|<1, r=1,2,3, \cdots)
$$

the $Y_{r}$ being independent normal variables with mean zero and variance $\sigma^{2}$.

For such a sequence of observations, constructing an S.P.R.T. of the simple hypothesis $m=m_{0}$ against the simple alternative $m=m_{1}$ when $\sigma^{2}$ and $\rho$ are known and deriving its sampling properties present no particular problems, since the logarithm of the likelihood ratio can be written as

where

$$
\log \frac{p\left(X_{0}, X_{1}, X_{2}, \cdots, X_{n} \mid m_{1}\right)}{p\left(X_{0}, X_{1}, X_{2}, \cdots, X_{n} \mid m_{0}\right)} \sim Z_{1}+Z_{2}+Z_{3}+\cdots+Z_{n}
$$

$$
Z_{r}=\frac{\left(m_{0}^{2}-m_{1}^{2}\right)(1-\rho)^{2}}{2 \sigma^{2}}+\frac{\left(m_{1}-m_{0}\right)(1-\rho)}{\sigma^{2}}\left(X_{r}-\rho X_{r-1}\right) .
$$

The $Z_{r}$ 's being mutually independent random variables, Wald's theory of sequential analysis of independent observations holds. The test is carried out by plotting $\sum_{r=1}^{n}\left(X_{r}-\rho X_{r-1}\right)$ against $n$. The same is true of a sequential test of $\sigma^{2}=\sigma_{0}^{2}$ against $\sigma^{2}=\sigma_{1}^{2}$ when $m$ and $\rho$ are known.

However, the test one would normally like to perform for the sequence $X_{0}$, $X_{1}, X_{2}, \cdots$ is about the parameter $\rho$. Consider, first, the case of the simple hypothesis $H_{0}: \rho=\rho_{0}$ (usually $\rho_{0}=0$ ) against $H_{1}: \rho=\rho_{1}\left(\rho_{1}>\rho_{0}\right.$, say), with known values of $m$ and $\sigma^{2}$, (here taken to be 0 and 1 respectively). We will assume that $X_{0}$ has the stationary distribution of the sequence. We then have the S.P.R.T. as follows: Continue sampling while 


$$
\log B<Z_{0}+Z_{1}+\cdots+Z_{n}<\log A,
$$

where

$$
\begin{aligned}
& Z_{0}=\frac{1}{2} \log \frac{1-\rho_{1}^{2}}{1-\rho_{0}^{2}}+\frac{\left(\rho_{1}^{2}-\rho_{0}^{2}\right) X_{0}^{2}}{2}, \\
& Z_{r}=\left(\rho_{1}-\rho_{0}\right) X_{r-1} X_{r}+\frac{\rho_{0}^{2}-\rho_{1}^{2}}{2} X_{r-1}^{2},
\end{aligned}
$$

and accept $H_{0}$ or $H_{1}$ according as the left-hand or the right-hand inequality is the first not satisfied. Relation (1.2) reduces to

$$
\begin{aligned}
\frac{1}{\rho_{1}-\rho_{0}}\left[\log B+\frac{1}{2} \log \frac{1-\rho_{0}^{2}}{1-\rho_{1}^{2}}\right] & <\sum_{r=1}^{n} X_{r-1} X_{r}-\frac{\rho_{0}+\rho_{1}}{2} \sum_{2}^{n} X_{r-1}^{2} \\
& <\frac{1}{\rho_{1}-\rho_{0}}\left[\log A+\frac{1}{2} \log \frac{1-\rho_{0}^{2}}{1-\rho_{1}^{2}}\right],
\end{aligned}
$$

and the test is carried out by plotting $\sum_{r=1}^{n} X_{r-1} X_{r}$ against $\sum_{r=2}^{n} X_{r-1}^{2}$. The constants $A$ and $B$ are given by, using Wald's [12] argument (which remains valid even if the observations are not independent), $A \sim\left(1-\varepsilon_{1}\right) / \varepsilon_{0}, B \sim \varepsilon_{1} /\left(1-\varepsilon_{0}\right)$, where $\varepsilon_{0}, \varepsilon_{1}$ are the probabilities of the first and second kinds of error respectively.

The sequence $\left\{Z_{r}\right\}$ is no longer independent, and to derive the O.C. and A.S.N. functions of the test one needs an analogue of Wald's Fundamental Identity for the sequence $\left\{Z_{r}\right\}$ defined on a Markov sequence $\left\{X_{r}\right\}$. An examination of the proof of the analogue for finite-state Markov chains given by Phatarfod [9], shows that the following generalization holds:

Let $X_{0}, X_{1}, X_{2}, \cdots$ be a Markov chain (discrete or continuous state space), on which is defined a sequence of random variables $\left\{Z_{r}\right\}, Z_{r}=h\left(X_{r-1}, X_{r}\right)$. Let $S_{N}$ denote the cumulative sum $Z_{0}+Z_{1}+Z_{2}+\cdots+Z_{N}$, and let $n$ be the least positive integer such that $S_{N}$ does not lie in the open interval $(b, a),(b<0, a>0)$. If the m.g.f. (for real $\theta$ in an interval $\left(\theta_{1}, \theta_{2}\right)$ around zero) of $S_{N}$ can be written for large $N$ as

$$
M_{N}(\theta) \sim C(\theta) \lambda_{1}^{N}(\theta)
$$

with $\lambda_{1}(0)=1, \lambda_{1}^{\prime \prime}(0)>\lambda_{1}^{\prime}(0)^{2}$, then

$$
E\left[\exp \left(\theta S_{n}\right) \lambda_{1}^{-n}(\theta) d\left(\theta \mid X_{n}\right)\right]=C(\theta),
$$

for all real $\theta$ in $\left(\theta_{1}, \theta_{2}\right)$ such that $\lambda_{1}(\theta) \geqq 1$. (The functions $C(\theta)$ and $d(\theta)$ have the relation $E\left[d\left(\theta \mid X_{0}\right)\right]=C(\theta)$.)

The proof is identical to the proof given in the earlier paper. The only requirement needed is that $P[n>N] \rightarrow 0$ as $N \rightarrow \infty$. This follows automatically, because (1.5) implies that $S_{N}$ is asymptotically normal with mean $N m=N \lambda_{1}^{\prime}(0)$ and variance $N \sigma^{2}=N\left[\lambda_{1}^{\prime \prime}(0)-\lambda_{1}^{\prime}(0)^{2}\right]$, and hence 


$$
\begin{aligned}
P[n>N] & \leqq \operatorname{Pr}\left[a<S_{N}<b\right] \\
& =\Phi\left(\frac{a-N m}{\sqrt{N \sigma}}\right)-\Phi\left(\frac{b-N m}{\sqrt{ } N \sigma}\right) \\
& \rightarrow 0 \quad \text { as } N \rightarrow \infty .
\end{aligned}
$$

A considerable amount of work has been done to establish conditions under which result (1.5) holds. The problem is essentially of generalizing results of Perron and Frobenius for non-negative matrices. The earliest such generalization seems to be due to Jentzsch [6] for integral operators with a positive kernel. More recently results have been given by Krein and Rutman [8], Birkhoff [1], Karlin [7], Chun [2], Harris [5] and Vere-Jones [11]. However, it is not immediately known how to determine the quantity $\lambda_{1}(\theta)$, the 'largest eigen value', and it seems to the present author, that this can be determined only by ad hoc methods.

As in the case of the analogue for finite state Markov chains, $\lambda_{1}(\theta)$ takes the role of the m.g.f. $M(\theta)$ in the independence case, and has the usual properties e.g. $\lambda_{1}^{\prime}(0)=E(Z)$ etc. Moreover, for the case considered above, it is found that $\lambda_{1}(\theta)$ is a convex function of $\theta$ having a minima near zero. This gives a unique non-zero real solution $\theta_{0}$ for $\lambda_{1}(\theta)=1$.

From the Identity (1.6), one can now readily obtain the usual results for the random walk process $\left\{S_{N}\right\}$. Unfortunately, except in special cases, only approximate expressions can be obtained. This is due to the fact that we ignore the excess of $S_{n}$ over the boundaries, and the terms $C(\theta)$ and $d\left(\theta \mid X_{n}\right)$ which depend on the distribution of $X_{0}$, and the value of $X_{n}$ respectively.

Putting $\theta=\theta_{0}$ (whenever such a value exists) in (1.6), the probability of the random walk terminating at $b$ before $a$ is given by,

$$
P_{b} \sim \frac{e^{a \theta_{0}}-1}{e^{a \theta_{0}}-e^{b \theta_{0}}} .
$$

The average duration of the random walk process is given by (in a manner similar to that in the case of finite state Markov chains (Phatarfod [10]),

$$
E(n) \sim \frac{b P_{b}+a P_{a}}{\lambda_{1}^{\prime}(0)}, \quad\left(\lambda_{1}^{\prime}(0) \neq 0\right) .
$$

In $\S 2$ we show that (1.5) holds for the Markov chain (1.1) with $Z_{r}$ 's as in (1.3). The O.C. and A.S.N. functions of the test are obtained from (1.7) and (1.8) respectively. In $\S 3$ we consider a Cox-type composite hypothesis test of $H_{0}: \rho=$ $\rho_{0}$ against $H_{1}=\rho=\rho_{1}$ when values of $m$ and $\sigma^{2}$ are not known.

\section{Test of the simple hypothesis $\rho=\rho_{0}$}

Consider the sequence $X_{0}, X_{1}, X_{2}, \cdots$ given by

$$
X_{r}=\rho X_{r-1}+Y_{r} \quad(|\rho|<1, r=1,2,3, \cdots)
$$


the $Y_{r}$ being independent normal variables with mean zero and variance unity. With the $Z_{r}$ 's as defined in (1.3), and $S_{N}=Z_{0}+Z_{1}+Z_{2}+\cdots+Z_{N}$, we have for real $\theta$,

$M_{N}(\theta)=E\left[\exp \left(\theta S_{N}\right)\right]=C \int \exp \left\{-\frac{1}{2}\left[x_{0}^{2}+x_{N}^{2}+a \sum_{r=1}^{N-1} x_{r}^{2}+2 b \sum_{r=1}^{N} x_{r} x_{r-1}\right]\right\}$

where

$$
\times d x_{0} d x_{1} \cdots d x_{N}
$$

$C=\left(\frac{1-\rho_{1}^{2}}{1-\rho_{0}^{2}}\right)^{\theta / 2} \cdot \frac{\left(1-\rho^{2}\right)^{\frac{1}{2}}}{(2 \pi)^{(n+1) / 2}}, \quad a=\theta\left(\rho_{1}^{2}-\rho_{0}^{2}\right)+\rho^{2}+1, \quad b=\left(\rho_{0}-\rho_{1}\right) \theta-\rho$.

The expression in the square brackets in the integrand is a quadratic form in $x_{0}, x_{1}, x_{2}, \cdots, x_{N}$ and hence, $M_{N}(\theta)$ may be written as (for values of $\theta$ such that $A_{N+1}$ is positive definite),

$$
M_{N}(\theta)=\left(\frac{1-\rho_{1}^{2}}{1-\rho_{0}^{2}}\right)^{\theta / 2} \frac{\left(1-\rho^{2}\right)^{\frac{1}{2}}}{\left|A_{N+1}\right|^{\frac{1}{2}}},
$$

where $A_{N+1}=\left(a_{i j}\right)$, the matrix of the quadratic form is given by

$$
\begin{array}{rlrl}
a_{i i} & =1(i=0, N), \quad a_{i i}=a(i \neq 0, N) ; & & (i, j=0,1,2, \cdots, N) \\
a_{i j}=b(|i-j|=1), \quad a_{i j}=0 & & \text { otherwise. }
\end{array}
$$

To determine $\left|A_{N+1}\right|$, we note

$$
\left|A_{N+1}\right|=\left|D_{N}\right|-b^{2}\left|D_{N-1}\right|,
$$

where $D$ is a matrix similar to $A$, with ' $a$ ' instead of 1 as the element in the first row and column. For $\left|D_{N}\right|$ we have

$$
\left|D_{N}\right|=a\left|D_{N-1}\right|-b^{2}\left|D_{N-2}\right|,
$$

with

$$
\left|D_{1}\right|=1, \quad\left|D_{2}\right|=a-b^{2} .
$$

From (2.2) and (2.3) we obtain,

$$
\left|D_{N}\right|=\frac{1}{\left(\mu_{1}-\mu_{2}\right)}\left[\left(1-\mu_{2}\right) \mu_{1}^{N}-\left(1-\mu_{1}\right) \mu_{2}^{N}\right],
$$

and hence

$$
\left|A_{N+1}\right|=\frac{1}{\left(\mu_{1}-\mu_{2}\right)}\left[\left(1-\mu_{2}\right)^{2} \mu_{1}^{N}-\left(1-\mu_{1}\right)^{2} \mu_{2}^{N}\right],
$$

where $\mu_{1}, \mu_{2}$ are the roots of the equation $x^{2}-a x+b^{2}=0$; we take

$$
\mu_{1}=\frac{1}{2}\left[a+\left(a^{2}-4 b^{2}\right)^{\frac{1}{2}}\right], \quad \mu_{2}=\frac{1}{2}\left[a-\left(a^{2}-4 b^{2}\right)^{\frac{1}{2}}\right] .
$$


The following properties of $\mu_{1}(\theta), \mu_{2}(\theta)$ can be easily verified:

(1) $\mu_{1}(\theta), \mu_{2}(\theta)$ are real and unequal for all $\theta$ in $\left(\theta_{1}, \theta_{2}\right)$ where

$$
\theta_{1}=-\frac{(1+\rho)^{2}}{\left(\rho_{1}-\rho_{0}\right)\left(2+\rho_{0}+\rho_{1}\right)}(<0), \quad \theta_{2}=\frac{(1-\rho)^{2}}{\left(\rho_{1}-\rho_{0}\right)\left(2-\rho_{0}-\rho_{1}\right)}(>0) .
$$

$\mu_{1}(\theta), \mu_{2}(\theta)$ are real and equal for $\theta=\theta_{1}, \theta_{2}$.

(2) $a>0$ for all $\theta$ in $\left(\theta_{1}, \theta_{2}\right)$, and hence $\mu_{1}(\theta)>0, \mu_{2}(\theta) \geqq 0, \mu_{1}(\theta)>\mu_{2}(\theta)$ for all $\theta$ in $\left(\theta_{1}, \theta_{2}\right)$.

(3) $\mu_{1}(\theta)$ is concave and $\mu_{2}(\theta)$ is convex in $\left(\theta_{1}, \theta_{2}\right)$.

(4) The equation $\mu_{1}(\theta)=1$ has two solutions $\theta=0$ and

$$
\theta=\theta_{0}=\frac{\rho_{0}+\rho_{1}-2 \rho}{\rho_{1}-\rho_{0}}, \quad\left(\rho \neq \frac{\rho_{0}+\rho_{1}}{2}\right),
$$

unless $\left|\rho-\rho_{0}-\rho_{1}\right|>1$, in which case $\theta_{0}=0$ is the only solution of $\mu_{1}(\theta)=1$. For $\left|\rho-\rho_{0}-\rho_{1}\right|=1$, the matrix $A_{N+1}$ is singular when $\theta=\theta_{0}$.

We will restrict $\theta$ in $\left(\theta_{1}, \theta_{2}\right)$ and the values of $\rho$ such that $\left|\rho-\rho_{0}-\rho_{1}\right|<1$. For such a range of values for $\theta$ and $\rho$, we have $\mu_{1}(\theta), \mu_{2}(\theta)$ real, $\mu_{1}(\theta)>0$, $0 \leqq \mu_{2}(\theta)<1, \mu_{1}(\theta)>\mu_{2}(\theta)$, and a non-zero real solution $\theta_{0}$ of $\mu_{1}(\theta)=1$, except when $\rho=\left(\rho_{0}+\rho_{1}\right) / 2$.

From (2.4) and (2.5) it can be seen that $\left|D_{r}\right|>0(r=1,2, \cdots, N)$ and $\left|A_{N+1}\right|>0$, and hence the matrix $A_{N+1}$ is positive definite. Further, from the properties of $\mu_{1}(\theta), \mu_{2}(\theta)$ given above, we have from (2.1), (2.5) as $N \rightarrow \infty$,

where

$$
M_{N}(\theta) \sim\left(\frac{1-\rho_{1}^{2}}{1-\rho_{0}^{2}}\right)^{\theta / 2} \frac{\left(1-\rho^{2}\right)^{\frac{1}{2}}\left(\mu_{1}-\mu_{2}\right)^{\frac{1}{2}}}{\left(1-\mu_{2}\right)} \cdot \mu_{1}(\theta)^{-N / 2}=C(\theta) \lambda_{1}^{N}(\theta)
$$

$$
\lambda_{1}(\theta)=\mu_{1}^{-\frac{1}{2}}(\theta) .
$$

It can be easily seen that $\left(\lambda_{1}^{\prime \prime}(0)-\lambda_{1}^{\prime}(0)^{2}\right)>0$, and hence the Identity $(1.6)$ and therefore results (1.7), (1.8) apply. We have the O.C. function as, (putting $a=$ $\log A, b=\log B)$,

$$
L(\rho) \sim \frac{A^{\theta(\rho)}-1}{A^{\theta(\rho)}-B^{\theta(\rho)}},
$$

where

$$
\theta(\rho)=\frac{\rho_{1}+\rho_{0}-2 \rho}{\rho_{1}-\rho_{0}}
$$

The limitation on the values of $\rho$ is not of serious consequence, as the range of permissible values of $\rho$ include the interval $\left(\rho_{0}, \rho_{1}\right)$ and beyond. It is interesting to note that the O.C. curve is identical to that of a test for the mean of a normal distribution in the independent observations case. 
For the A.S.N. we have,

$$
E_{\rho}(n)=\frac{2[L(\rho) \log B+(1-L(\rho)) \log A]}{-\mu_{1}^{\prime}(0)}, \quad\left(\rho \neq \frac{\rho_{0}+\rho_{1}}{2}\right) .
$$

Note

$$
-\mu_{1}^{\prime}(0) / 2=E_{\rho}(Z)=\frac{1}{2\left(1-\rho^{2}\right)}\left[\left(\rho_{0}^{2}-\rho_{1}^{2}\right)+2\left(\rho_{1}-\rho_{0}\right)\right]
$$

For $\rho=\left(\rho_{0}+\rho_{1}\right) / 2$, a formula can be obtained by differentiating (1.6) twice w.r.t. ' $\theta$ ' and putting $\theta=0$. Also since $\theta(\rho)$ and $L(\rho)$ are monotonic functions of $\rho$, the test given above can be used to test composite hypotheses of the form $\rho \leqq \rho^{\prime}$ against $\rho>\rho^{\prime}$, where $m$ and $\sigma^{2}$ are known.

\section{Test of composite hypothesis $\rho=\rho_{0}$}

We will now consider the general model given by (1.1) and derive a Cox-type (Cox [3]) test of the composite hypothesis $H_{0}: \rho=\rho_{0}$ against $H_{1}: \rho=\rho_{1}$ $\left(\rho_{1}>\rho_{0}\right)$.

The likelihood of the observations $X_{0}, X_{1}, X_{2}, \cdots, X_{n}$ assuming $X_{0}$ has the stationary distribution of the sequence is given by

$$
\begin{aligned}
p\left(x_{0}, x_{1}, \cdots, x_{n}\right)=\text { const } \cdot \exp \left\{-\frac{1}{2 \sigma^{2}}\right. & {\left[\left(x_{0}-m\right)^{2}+\left(x_{n}-m\right)^{2}+\left(1+\rho^{2}\right)\right.} \\
& \left.\left.\times \sum_{r=1}^{n-1}\left(x_{r}-m\right)^{2}-2 \rho \sum_{r=1}^{n}\left(x_{r-m}\right)\left(x_{r-1}-m\right)\right]\right\}
\end{aligned}
$$

Writing

$$
\begin{aligned}
\vec{x} & =\left(\frac{1}{2} x_{0}+x_{1}+x_{2}+\cdots \frac{1}{2} x_{n}\right) / n, \\
S^{2} & =\frac{1}{2}\left(x_{0}-\bar{x}\right)^{2}+\sum_{r=1}^{n-1}\left(x_{r}-\bar{x}\right)^{2}+\frac{1}{2}\left(x_{n}-\bar{x}\right)^{2},
\end{aligned}
$$

and

$$
r_{n}=\sum_{r=1}^{n}\left(x_{r}-\bar{x}\right)\left(x_{r-1}-\bar{x}\right) / S^{2}
$$

the expression in the square brackets above can be written as

$$
\frac{\left(1-\rho^{2}\right)}{2}\left\{\left(x_{0}-m\right)^{2}+\left(x_{n}-m\right)^{2}\right\}+\left(1+\rho^{2}\right) S^{2}-2 \rho r_{n} S^{2}+n(\bar{x}-m)^{2}(1-\rho)^{2}
$$

The quantities $x_{0}, x_{n}, \bar{x}, S^{2}$ and $r_{n}$, therefore form a set of jointly sufficient statistics for the parameters $m, \sigma^{2}$ and $\rho$. For large $n$ we may ignore $x_{0}$ and $x_{n}$ and conclude that $\bar{x}, S^{2}$ and $r_{n}$ are asymptotically jointly sufficient for $m, \sigma^{2}$ and $\rho$. Moreover, the distribution of $r_{n}$ as given by Daniels [4] is independent of $m$ and $\sigma^{2}$. Furthermore the transformation $y_{i}=a x_{i}+b((i=0, \cdots, n)$ satisfies condition (iv) of Cox' 
Theorem. Hence a test can be constructed based on the 'observations' $r_{2}, r_{3}, \cdots$, $r_{n}$. The asymptotic distribution of $r_{n}$ as given by Daniels has the p.d.f. (ignoring terms of order $O\left(n^{-\frac{3}{2}}\right)$ ),

$$
L\left(r_{n}\right) \sim \frac{\Gamma\left(\frac{1}{2} N+\frac{3}{2}\right)}{2 \pi^{\frac{1}{2}} \Gamma\left(\frac{1}{2} N\right)[N(1-\rho)-(1+\rho)]} \frac{\left(1-r_{n}\right)\left(1-r_{n}^{2}\right)^{\frac{1}{2} N-1}}{\left(1-2 \rho r_{n}+\rho^{2}\right)^{\frac{1}{2}(N-1)}}
$$

where

$$
N=n+\frac{\rho^{2}}{\left(1-\rho^{2}\right)} \text {. }
$$

The test can be written: Continue sampling while

$$
\log B<\log \frac{L\left(r_{n} \mid \rho_{1}\right)}{L\left(r_{n} \mid \rho_{0}\right)}<\log A
$$

Substituting $L\left(r_{n}\right)$ from (3.1) in (3.2), using Stirling's approximation, and ignoring terms $O\left(n^{-2}\right)$, the middle term in (3.2) reduces to

$$
\begin{aligned}
\log \frac{1-\rho_{0}}{1-\rho_{1}} & +\frac{\frac{5}{2}\left(\rho_{1}^{2}-\rho_{0}^{2}\right)+2\left(\rho_{1}-\rho_{0}\right)\left(1+\rho_{0} \rho_{1}\right)}{n\left(1-\rho_{1}^{2}\right)\left(1-\rho_{0}^{2}\right)} \\
& +\frac{1}{2}\left(N_{0}-1\right) \log \left(1-2 \rho_{0} r_{n}+\rho_{0}^{2}\right)-\frac{1}{2}\left(N_{1}-1\right) \log \left(1-2 \rho_{1} r_{n}+\rho_{1}^{2}\right) \\
& +\left(\frac{N_{1}-N_{0}}{2}\right) \log \left(1-r_{n}^{2}\right),
\end{aligned}
$$

where

$$
N_{i}=n+\frac{\rho_{i}^{2}}{1-\rho_{i}^{2}}, \quad(i=0,1) .
$$

For fairly large $n$, we have (3.2) approximately as

$$
\begin{aligned}
\log \frac{B\left(1-\rho_{1}\right)}{\left(1-\rho_{0}\right)}< & \frac{\frac{5}{2}\left(\rho_{1}^{2}-\rho_{0}^{2}\right)+2\left(\rho_{1}-\rho_{0}\right)\left(1+\rho_{0} \rho_{1}\right)}{n\left(1-\rho_{1}^{2}\right)\left(1-\rho_{0}^{2}\right)} \\
& +\frac{n}{2} \log \frac{\left(1-2 \rho_{0} r_{n}+\rho_{0}^{2}\right)}{\left(1-2 \rho_{1} r_{n}+\rho_{1}^{2}\right)}<\log \frac{A\left(1-\rho_{1}\right)}{\left(1-\rho_{0}\right)},
\end{aligned}
$$

from which acceptance numbers $r_{n}^{+}$and rejection numbers $r_{n}^{-}$may be calculated.

\section{Acknowledgement}

I am grateful to Professor M. S. Bartlett for suggesting the problem to me. 


\section{References}

[1] G. Birkhoff, 'Extensions of Jentzsch's theorem', Trans. Amer. Math. Soc. 85 (1957), 219227.

[2] K. L. Chung, Markov chains with stationary transition probabilities (Springer-Verlag, Berlin, 1960).

[3] D. R. Cox, 'Sequential tests for composite hypotheses', Proc. Camb. Phil. Soc. 48 (1952), 290-299.

[4] H. E. Daniels, 'The approximate distribution of serial correlation coefficients', Biometrika 43 (1956), 169-185.

[5] T. E. Harris, The Theory of Branching Processes (Springer-Verlag, Berlin, 1963).

[6] R. Jentzsch, 'Über Integralgleichungen mit positivem Kern', J. Reine Angew, Math. 141 (1912), 235-244.

[7] S. Karlin, 'Positive Operators', J. Math. Mech. 8 (1957), 907-938.

[8] M. G. Krein and M. A. Rutman, 'Linear operators leaving invariant a cone in a Banach space', Amer. Math. Soc. Translation No. 26, 1950.

[9] R. M. Phatarfod, 'Sequential analysis of dependent observations I', Biometrika 52 (1965), 157-165.

[10] R. M. Phatarfod, 'A note on the cumulation sums of Markovian variables', J. Aust. Math. Soc. 5 (1965), 285-287.

[11] D. Vere-Jones, 'Ergodic properties of non-negative matrices-I', Pacific. J. Math. 22 (1967) $361-386$.

[12] A. Wald, Sequential Analysis (J. Wiley, New York, 1947).

\section{Monash University \\ Clayton, Victoria}

